\title{
Involvement of retinoblastoma-associated protein 48 during photodynamic therapy of cervical cancer cells
}

\author{
SHUXIA WU ${ }^{1,2}$, LIJUN WANG $^{3}$, XINGYE REN $^{2}$, YULU PAN $^{2}$, YAN PENG $^{2}$, \\ XIAOYAN ZOU ${ }^{2}$, CUIGE $\mathrm{SHI}^{4}$ and YOUZHONG ZHANG ${ }^{1}$ \\ ${ }^{1}$ Department of Gynecology and Obstetrics, Qilu Hospital of Shandong University, Jinan, \\ Shandong 250012; ${ }^{2}$ Department of Gynecology and Obstetrics, The Fifth People's Hospital of Jinan; \\ ${ }^{3}$ Department of Pediatrics, Shandong Provincial Hospital Affiliated to Shandong University, Jinan, \\ Shandong 250021; ${ }^{4}$ National Research Institute of Family Planning, Beijing 100081, P.R. China
}

Received November 14, 2015; Accepted November 24, 2016

DOI: $10.3892 / \mathrm{mmr} .2017 .6151$

\begin{abstract}
Aminolevulinic acid-mediated photodynamic therapy (ALA-PDT) is an effective treatment option for cervical intraepithelial neoplasia, the precancerous lesion of cervical cancer, and early cervical cancer, particularly for young or nulliparous women who want to remain fertile. A previous report described the involvement of histone deacetylases (HDAC) during ALA-PDT mediated apoptosis in the cerebral cortex of a mouse model. Retinoblastoma-associated protein 48 (RbAp48), a highly abundant component of HDACs, is a critical mediator that controls the transforming activity of human papillomavirus 16 in cervical cancer cells. The aim of the present study was to investigate the involvement of RbAp48 in ALA-PDT-induced cell death in cervical cancer cells. RbAp48 was significantly upregulated in cervical cancer cell lines treated with ALA-PDT, including SiHa and HeLa cells. To establish the relevance of RbAp48 and the efficacy of ALA-PDT in cervical cancer cells, the effect of ALA-PDT was investigated in SiHa or HeLa cells following the depletion of RbAp48 by small interfering RNA (siRNA). Reduction of $\mathrm{RbAp} 48$ led to the reduced suppression of proliferation and apoptosis induced by ALA-PDT in cervical cancer cells, which was associated with a reduction in tumor suppressor protein 53 (p53), retinoblastoma ( $\mathrm{Rb}$ ), apoptosis-related enzyme caspase- 3 , and increased levels of the oncogenic genes, human papillomavirus E6 and E7. These results provide evidence that RbAp48 is an important contributor to the efficacy of ALA-PDT in cervical cancer cells. RbAp48 may be a therapeutic target that may help to improve the treatment of cervical cancer.
\end{abstract}

Correspondence to: Professor Youzhong Zhang, Department of Gynecology and Obstetrics, Qilu Hospital of Shandong University, 107 Wenhua Xi Road, Jinan, Shandong 250012, P.R. China E-mail: zhangyouzhong@sdu.edu.cn

Key words: cervical cancer, photodynamic therapy, apoptosis retinoblastoma-associated protein 48

\section{Introduction}

Cervical cancer is one of the major causes of cancer morbidity and mortality in women globally, particularly in developing countries. According to the World Health Organization International Agency for Research on Cancer (GLOBOCAN 2012), >500,000 women worldwide were diagnosed with cervical carcinoma each year, of which $>80 \%$ were in less developed countries (1). In China, cervical cancer cases are increasingly prevalent in young patients (2). Currently, the established treatment for cervical cancer, particularly in the early stages, is resection and radiotherapy. However, these mainstream approaches may be associated with subsequent pregnancy complications, which is problematic particularly for young or nulliparous women who wish to remain fertile. Improvements in treatment are required for patients with cervical cancer and photodynamic therapy (PDT), a treatment that preserves the cervix, may be a suitable option.

PDT is an innovative technique that is widely used for local treatment of various solid tumors (3-5) and certain non-malignant diseases (6-8). PDT involves using a specific wavelength of light to activate a photosensitizer that is selectively accumulated in the target tissue. PDT causes destruction of stained cells through the production of singlet oxygen or superoxide, and induces irreversible cell damage through direct and indirect cytotoxicity (9). 5-Aminolaevulanic acid (ALA)-mediated PDT (ALA-PDT) is an approved therapeutic option for local therapy of various human precancerous and cancerous lesions with encouraging clinical outcomes (10). However, its specific mechanisms are not fully understood and its efficacy is remarkably varied. A complete understanding of the molecular mechanisms of PDT-mediated cell destruction may lead to improvements in its therapeutic efficacy. Epigenetic regulation of transcription and histone modification might be involved in PDT-mediated cell destruction (11). The upregulation of the histone deacetylases HDAC-1 and HDAC-11 has also been demonstrated in the mouse cerebral cortex following ALA-PDT (11). Retinoblastoma-associated protein 48 (RbAp48), a highly abundant component of HDACs (12), is required for negative regulation of E2F transcription factor activity through its interaction with HDAC1 
and HDAC3 (13). A previous study has also demonstrated that $\mathrm{RbAp} 48$ is a critical mediator controlling human papilloma virus (HPV)-16 transforming activity in HPV-induced cervical carcinogenesis (14). RbAp48 suppresses the growth of cervical cancer and reverts transformed phenotypes of cervical cancer in vitro and in vivo. Furthermore, RbAp48 was identified as a radiosensitive gene in a microarray analysis used for selecting radiosensitivity prediction molecules (15). Finally, another previous study indicated the involvement of $\mathrm{RbAp} 48$ in radiation-response and overexpression of RbAp48 enhanced the radiosensitivity of cervical cancer (16).

The present study demonstrated that PDT induces RbAp48, which leads to cancer cell destruction through upregulation of tumor suppressor protein 53 (p53) and retinoblastoma protein $(\mathrm{Rb})$ expression, and downregulation of the HPV genes, E6 and E7. The current study provides evidence for the involvement of RbAp48 in anti-proliferative responses to ALA-PDT.

\section{Materials and methods}

Cell culture and PDT. SiHa and HeLa cervical cancer cells were obtained from the American Type Culture Collection (Manassas, VA, USA) and cultured in Dulbecco's modified Eagle's medium (Invitrogen; Thermo Fisher Scientific, Inc., Waltham, MA, USA) supplemented with $10 \%$ fetal bovine serum (FBS; Invitrogen; Thermo Fisher Scientific, Inc.), under standard culture conditions. Cells, 80-90\% confluent, were used in all experiments. ALA hydrochloride, obtained from Sigma-Aldrich (Merck Millipore; Darmstadt, Germany), was dissolved in DMEM. Various concentrations $(0,50$, 100 and $150 \mu \mathrm{M})$ of ALA dissolved in DMEM were added to cells, which were then incubated for $6 \mathrm{~h}$ in the dark at $37^{\circ} \mathrm{C}$ and $5 \% \mathrm{CO}_{2}$. Following administration of ALA, all samples went through 3 washing steps with PBS. Fresh culture medium (DMEM supplemented with 10\% FBS) was then added to each well, followed by light-emitting diode laser irradiation (Wuhan Yage Optic and Electronic Technology Co., Ltd., Wuhan, China) at a wavelength of $630 \mathrm{~nm}$ for $5 \mathrm{~min}$ and the laser energy was $10 \mathrm{~J} / \mathrm{cm}^{2}$. Then, cells were incubated in the dark at $37^{\circ} \mathrm{C}, 5 \% \mathrm{CO}_{2}$ for $24 \mathrm{~h}$.

Cell viability and cell counting assay. Cells were seeded $2 \times 10^{3}$ per well in a 96-well culture plate for the cell viability assay and $1 \times 10^{4}$ per well in 24 -well plates for the cell counting assay. Cells were then treated with various concentrations $(0,50,100$ or $150 \mu \mathrm{M})$ of ALA. Cell viability was evaluated by Cell Counting Kit-8 (Dojindo Molecular Technologies, Inc., Kumamoto, Japan) according to the manufacturer's protocol as described, $24 \mathrm{~h}$ after ALA-PDT treatment. For the cell counting assay, cells were treated as above and detected as described previously (14). Experiments were performed in triplicate to ensure the reproducibility of the results. And the half-maximal inhibitory concentration was derived from the dose-response curve.

Western blotting. Cells were harvested $24 \mathrm{~h}$ after ALA-PDT treatment and then washed 3 times with cold PBS. Total protein was extracted from cells by the use of NP-40 lysis buffer (Beyotime Institute of Biotechnology, Haimen, China) according to the manufacturer's protocol, separated on
$12 \%$ polyacrylamide-SDS gels and transferred onto a $0.2 \mu \mathrm{m}$ polyvinylidene fluoride membrane (Immobilon ${ }^{\circledR}$; EMD Millipore, Billerica, MA, USA). After blocking with TBS $(0.1 \%$ Tween-20) containing 5\% non-fat milk for $1 \mathrm{~h}$ at room temperature, the blot was washed with TBST (10 mM TBS, $100 \mathrm{mM} \mathrm{NaCl}, 0.1 \%$ Tween-20) 3 times for $10 \mathrm{~min}$. The blot was then incubated with mouse anti-RbAp48 antibodies (1:1,000; cat. no. ab55778; Abcam, Cambridge, UK) or glyceraldehyde phosphate dehydrogenase antibodies (GAPDH; 1:2,000; cat. no. ab8245; Abcam, UK) overnight at $4^{\circ} \mathrm{C}$. The blot was washed again 3 times for $10 \mathrm{~min}$ and then incubated with a goat anti-mouse horseradish peroxidase-conjugated secondary antibody $(1: 1,000$; cat. no. ab6789; Abcam) for $1 \mathrm{~h}$ at room temperature. The blot was washed 3 times for $10 \mathrm{~min}$ and immune complexes were visualized by chemiluminescence using an ECL kit (GE Healthcare Life Sciences, Chalfont, UK) and imaged using ImageQuant LAS 4000 (GE Healthcare Life Sciences). The images were analyzed and quantified using Image J software version 1.44 (National Institutes of Health, Bethesda, MD, USA). The intensity of bands was normalized in relative to GAPDH signals. Each experiment was repeated 3 times.

Reverse transcription-quantitative polymerase chain reaction $(R T-q P C R)$. Total RNA was extracted from cells with TRIzol reagent (Invitrogen; Thermo Fisher Scientific, Inc.), and then $5 \mu \mathrm{g}$ total RNA was purified and reversely transcribed to cDNA using random primers with the PrimeScript ${ }^{\mathrm{TM}}$ RT Reagent Kit with gDNA Eraser (Takara Bio, Inc., Otsu, Japan) following the manufacturer's protocol. Amplification of PCR products was quantified using SYBR ${ }^{\circledR}$ Premix Ex Taq ${ }^{\mathrm{TM}}$ (Takara Bio, Inc., Otsu, Japan) and performed on the LightCycler 2.0 Instrument (Roche Diagnostics, Basel, Switzerland) equipped with a LightCycler Software version 4.0 (Roche Diagnostics, Basel, Switzerland) using the following PCR conditions: 40 cycles, $95^{\circ} \mathrm{C}$ for $15 \mathrm{~s}, 60^{\circ} \mathrm{C}$ for $1 \mathrm{~min}$. Primers used for RT-qPCR were as follows (forward and reverse, respectively): RbAp48, forward 5'-ATGCCCCAGAACCCTTGTATC-3' and reverse 5'-GCCCATAGCCTTCCTTCTGAT-3'; HPV-16 E6, forward 5'-AATGTTTCAGGACCCACAGG-3' and reverse 5'-TCA CGTCGCAGTAACTGTTG-3'; HPV-16 E7, forward 5'-AGT GTGACTCTACGCTTCGG-3' and reverse 5'-TGTGCCCAT TAACAGGTCTT-3'; p53, forward 5'-GGCAGCTGGTTA GGTAGAGG-3' and reverse 5'-AGGTCGACCAAGAGGTTG TC-3'; Rb, forward 5'-ACCCAGAAGCCATTGAAATC-3' and reverse 5'-TCTGGGTGCTCAGACAGAAG-3'; capase-3, forward 5'-TAAATGAATGGGCTGAGCTG-3' and reverse 5'-ATGGAGAAATGGGCTGTAGG-3'; $\beta$-actin, forward 5'-CTCCAAATGCAAACTGGATG-3' and reverse 5'-TGT TGATTTGGGCACAGACT-3', as described previously (14). Target gene expression levels were normalized to $\beta$-actin levels in the same reaction. The $\Delta \Delta \mathrm{Cq}$ method was used to normalize mRNA levels (17).

Small interfering RNA (siRNA) transfection. Cells were plated in 6-well culture plates at $1 \times 10^{5}$ cells/well and incubated at $37^{\circ} \mathrm{C}$ until they reached $80 \%$ confluency. Lipofectamine ${ }^{\circledR} 2000$ reagent (Invitrogen, Thermo Fisher Scientific, Inc.) was used to transfect siRNA oligonucleotides that targeted RbAp48 (siRbAp48; Sigma-Aldrich; Merck Millipore) and mammalian 
expression pSUPER vector (OligoEngine, Seattle, WA, USA) into $\mathrm{SiHa}$ and HeLa cells as described previously (14). The sequences of siRNAs were as follows: 5'-CAGGGCATACGG CAGTAGT-3' for si-RbAp48 (1) and 5'-CGAGGAATACAA AATATGG-3' for si-RbAp48 (2), as previously described (14). Cells were transfected with the pSUPER vector to generate a control line. The sequences of control siRNA contained in the pSUPER vector was 5'-GACTCCAGTGGTAATCTAC-3'. At $48 \mathrm{~h}$ post-transfection, cells were harvested and used for further experiments. Expression of RbAp48 was determined by RT-qPCR and western blot assay.

Apoptosis rate analysis by flow cytometry. Cells were plated in $35 \mathrm{~mm}$ dishes and then received $100 \mu \mathrm{M}$ ALA-PDT treatment or irradiation as described earlier, and were incubated at $37^{\circ} \mathrm{C}$ in the dark for $24 \mathrm{~h}$. Then, the medium was removed, cells were washed twice with PBS and collected in tubes $\left(1 \times 10^{6}\right)$ and were then stained using an Annexin V-FITC/PI Apoptosis Detection kit (BestBio, Co., Shanghai, China) according to the manufacturer's protocols. Analysis was carried out at activating wavelength $488 \mathrm{~nm}$ and fluorescence emission was monitored at $623 \mathrm{~nm}$ by a FACS Calibur flow cytometer (BD Biosciences, Franklin Lakes, NJ, CA, USA) and the data analyzed using by FACS Calibur flow cytometer instrument equipped with BD CellQuest Pro software version 5.1 (BD Biosciences).

Statistical analysis. Data are presented as the mean \pm standard deviation. Statistical comparisons between means were assessed by Student's t-test or two-way analysis of variance using GraphPad Prism (version 6.0; GraphPad Software, Inc., La Jolla, CA, USA). Bonferroni's post hoc test was used for post hoc comparison. $\mathrm{P}<0.05$ was considered to indicate a statistically significant difference.

\section{Results}

ALA-PDT induces RbAp48 expression in SiHa and HeLa cells. To clarify the function of RbAp48 in ALA-PDT treatment, the present study initially determined the expression pattern of RbAp48. mRNA and protein levels of RbAp48 were significantly upregulated in $\mathrm{SiHa}$ and HeLa cells subjected to all concentrations of ALA-PDT treatment, compared with untreated cells $(\mathrm{P}<0.05$; Fig. 1). A previous study verified that RbAp48 controls the transforming activity of HPV-16 in cervical cancer (14). Therefore, RbAp48 may be involved in the response of cervical cancer cells to ALA-PDT treatment.

Inhibition of RbAp48 expression via siRNA-mediated silencing attenuates reduced viability caused by ALA-PDT in SiHa and HeLa cells. To verify whether altered expression of RbAp48 affects proliferation of PDT-surviving cervical cancer cells, the current study initially suppressed RbAp48 gene expression in $\mathrm{SiHa}$ and HeLa cells using siRbAp48, and subsequently performed PDT on cells and evaluated the cell viability. As demonstrated in Fig. 2A, siRbAp48 inhibited protein expression of RbAp48 compared with pSUPER vector control (si-control) and parent $\mathrm{SiHa}$ or HeLa cells. However, siRbAp48-2 had a stronger effect. Subsequently, the effect of reduced RbAp48 expression on cellular responses to ALA-PDT in SiHa and HeLa cells by siRNA knockdown
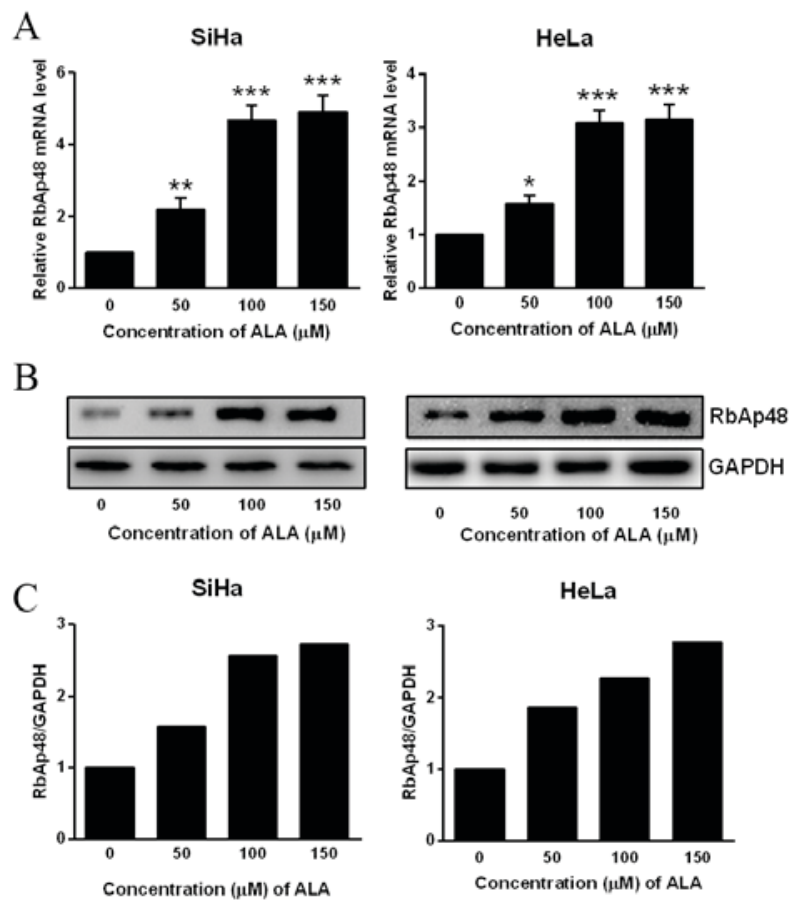

Figure 1. ALA-PDT induces the expression of RbAp48 in cervical cancer cell lines. (A) Relative mRNA levels of RbAp48 in SiHa and HeLa cells assayed by reverse transcription-quantitative polymerase chain reaction. Expression of RbAp48 in each sample was normalized to that of $\beta$-actin. Data are presented as the mean + standard deviation of 3 independent experiments. (B) Protein levels of RbAp48 in SiHa and HeLa cells were assayed by western blot with anti-RbAp48 antibody $24 \mathrm{~h}$ after ALA-PDT treatment. GAPDH was a loading control. Data from a typical experiment repeated 3 times with similar results are presented. (C) The ratio of RbAp48/GAPDH for each was analyzed by densitometry and presented as bar graphs. ( $\mathrm{P}<0.05$; ${ }^{* *} \mathrm{P}<0.01 ;{ }^{* * *} \mathrm{P}<0.001$ vs. $0 \mu \mathrm{M}$ ALA). RbAp48, Retinoblastoma-associated protein; 48ALA, 5-aminolevulinic acid; ALA-PDT, ALA-mediated photodynamic therapy.

of RbAp48 was investigated. The CCK-8 assay (Fig. 2B) and cell counting assay (Fig. 2C) revealed that reduced RbAp48 impaired ALA-PDT-induced cell growth inhibition in $\mathrm{SiHa}$ and HeLa cervical cancer cell lines compared with si-control cells $(\mathrm{P}<0.05)$.

RbAp48 modulates ALA-PDT-induced apoptosis. The anti-tumor effect of ALA-PDT is predominantly mediated via apoptosis. Therefore, the current study investigated whether RbAp48 has a role in PDT-induced apoptosis. Knockdown of $\mathrm{RbAp} 48$ in SiHa and HeLa cells led to a significant reduction in PDT-induced apoptosis compared with si-control vector groups $(\mathrm{P}<0.001$ and $\mathrm{P}<0.001$, respectively; Fig. 3).

RbAp48 regulates $m R N A$ expression of tumor suppressors, apoptosis-associated enzymes and oncogenic viral genes in cervical cancer cells in response to ALA-PDT. To elucidate the molecular mechanism by which RbAp48 functions in the response of cervical cancer cells to ALA-PDT, RT-qPCR was performed. mRNA expression changes of tumor suppressors $\mathrm{Rb}$ and $\mathrm{p53}$, apoptosis-associated enzyme caspase-3, and HPV oncogenes, E6 and E7, in cervical cancer cell lines were investigated. As presented in Fig. 4, silencing RbAp48 significantly reduced mRNA levels of tumor suppressors p53 $(\mathrm{SiHa}$, $\mathrm{P}<0.001$; HeLa, $\mathrm{P}<0.001$; Fig. 4A) and $\mathrm{Rb}(\mathrm{SiHa}, \mathrm{P}<0.01$; 
A

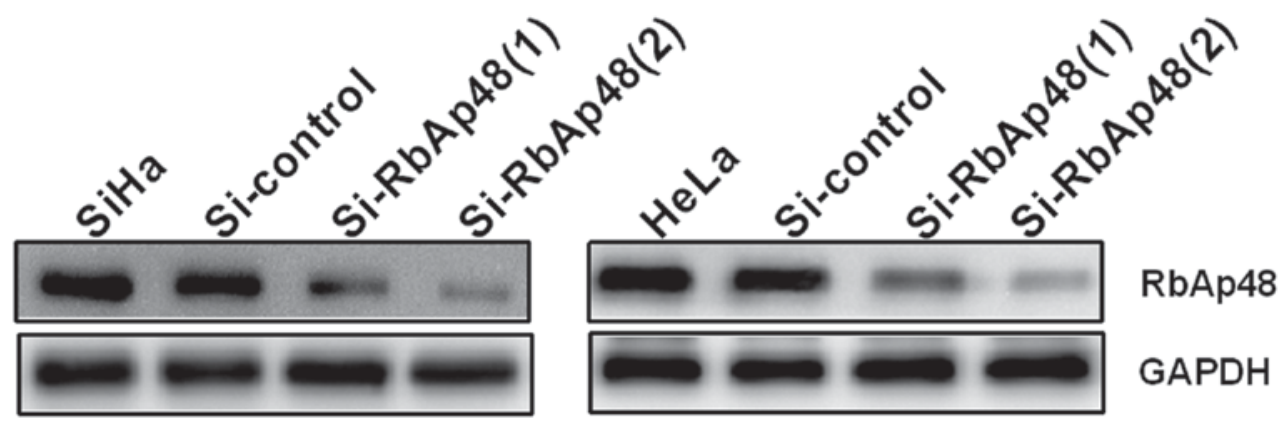

$\mathrm{B}$
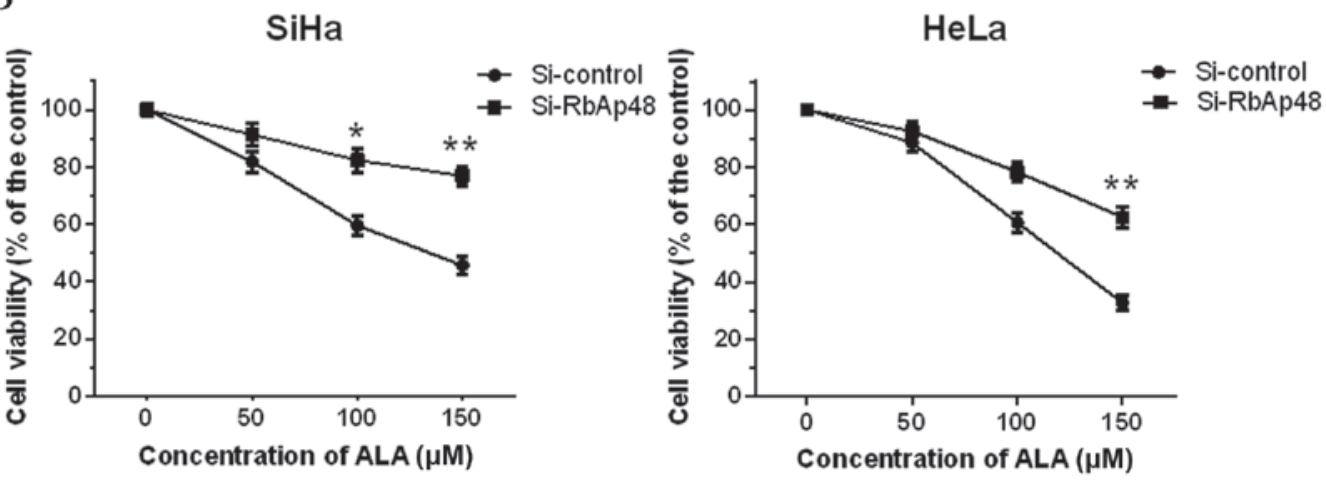

$\mathrm{C}$

$\mathrm{SiHa}$

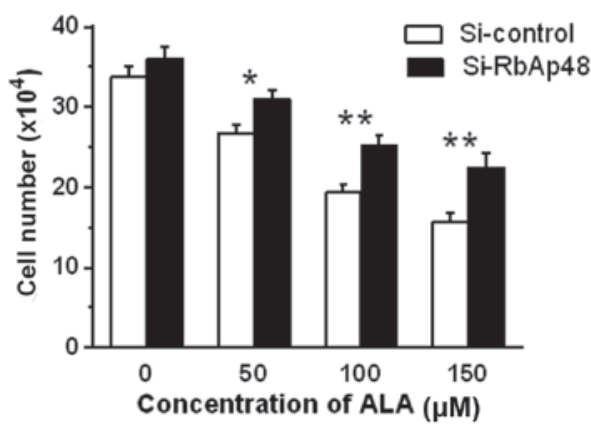

HeLa

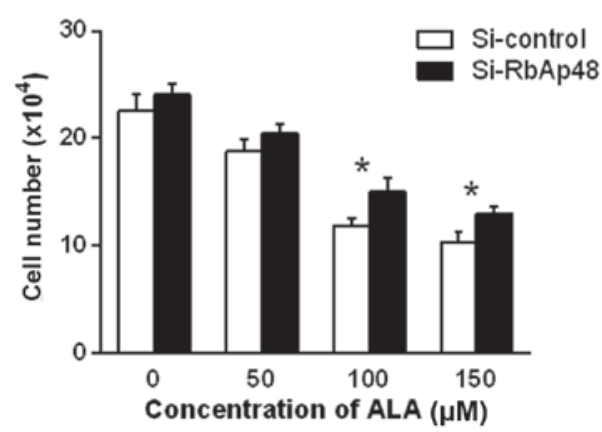

Figure 2. Knockdown of RbAp48 in SiHa and HeLa cells reduces anti-proliferative effects of ALA-PDT treatment. (A) RbAp48 protein expression is reduced in the si-RbAp48 1 and 2 transfected cells compared with the respective control cells. Si-RbAp48 reduced cell growth inhibition induced by ALA-PDT on $\mathrm{SiHa}$ and HeLa cervical cancer lines, as measured by (B) Cell Counting Kit-8 and (C) cell counting assay. Data are presented as the mean \pm standard deviation of 3 independent experiments. ( $\mathrm{P}<0.05 ;{ }^{* *} \mathrm{P}<0.01$ vs. si-control group). ALA-PDT, ALA-mediated photodynamic therapy; $\mathrm{Si}$, small interfering RNA; RbAp48, retinoblastoma-associated protein 48; ALA, 5-aminolevulinic acid.

HeLa, $\mathrm{P}<0.05$; Fig. 4B) induced by ALA-PDT treatment in cervical cancer cells compared with the si-control treatments. Similar results were observed for apoptosis-associated enzyme caspase-3 (SiHa, P<0.001; HeLa, P<0.01; Fig. 4C). Notably, siRNA knockdown of RbAp48 also significantly increased the E6 (SiHa, $\mathrm{P}<0.01$; HeLa, $\mathrm{P}<0.001$; Fig. 4D) and E7 ( $\mathrm{SiHa}$, $\mathrm{P}<0.01$; HeLa, $\mathrm{P}<0.05$; Fig. 4E) levels, that were reduced by PDT, which have important roles in HPV-induced cervical cancer. Therefore, RbAp48-mediated sensitivity to ALA-PDT treatment in cervical cancer cells may be due, at least partially, to its regulation of the expression of tumor suppressors p53 and $\mathrm{Rb}$, apoptosis-associated enzyme caspase-3, and the HPV oncogenes, E6 and E7.

\section{Discussion}

PDT, which has curative effects and causes remarkably little scarring, has been clinically approved and has distinct advantages in its ability to locally treat cervical cancer and cervical intraepithelial neoplasia (CIN; the precancerous lesion of cervical cancer) $(3,5,8,18)$. Various clinical studies have revealed that PDT is of particular value to women with CIN or early cervical cancer who are interested in having children later in life $(19,20)$. Furthermore, PDT, as an ideal adjuvant therapy (4), may be applied prior to or following chemotherapy or surgery and can be repeated as many times as necessary without cumulative toxicity $(10,21,22)$. However, in clinical trials, tumor responses induced by PDT varied between individual patients. In certain patients, a number of tumor cells may become resistant to PDT and survive, therefore leading to relapse and metastasis $(3,5,18)$. RbAp48, a potential therapeutic target in cervical cancer (14), is a radiation-inducible gene that has the potential to increase the sensitivity of cervical cancer cells to radiation (16). In the present study, mRNA and protein levels of RbAp48 were upregulated in two cervical cancer cell lines that had been subjected to ALA-PDT. To 


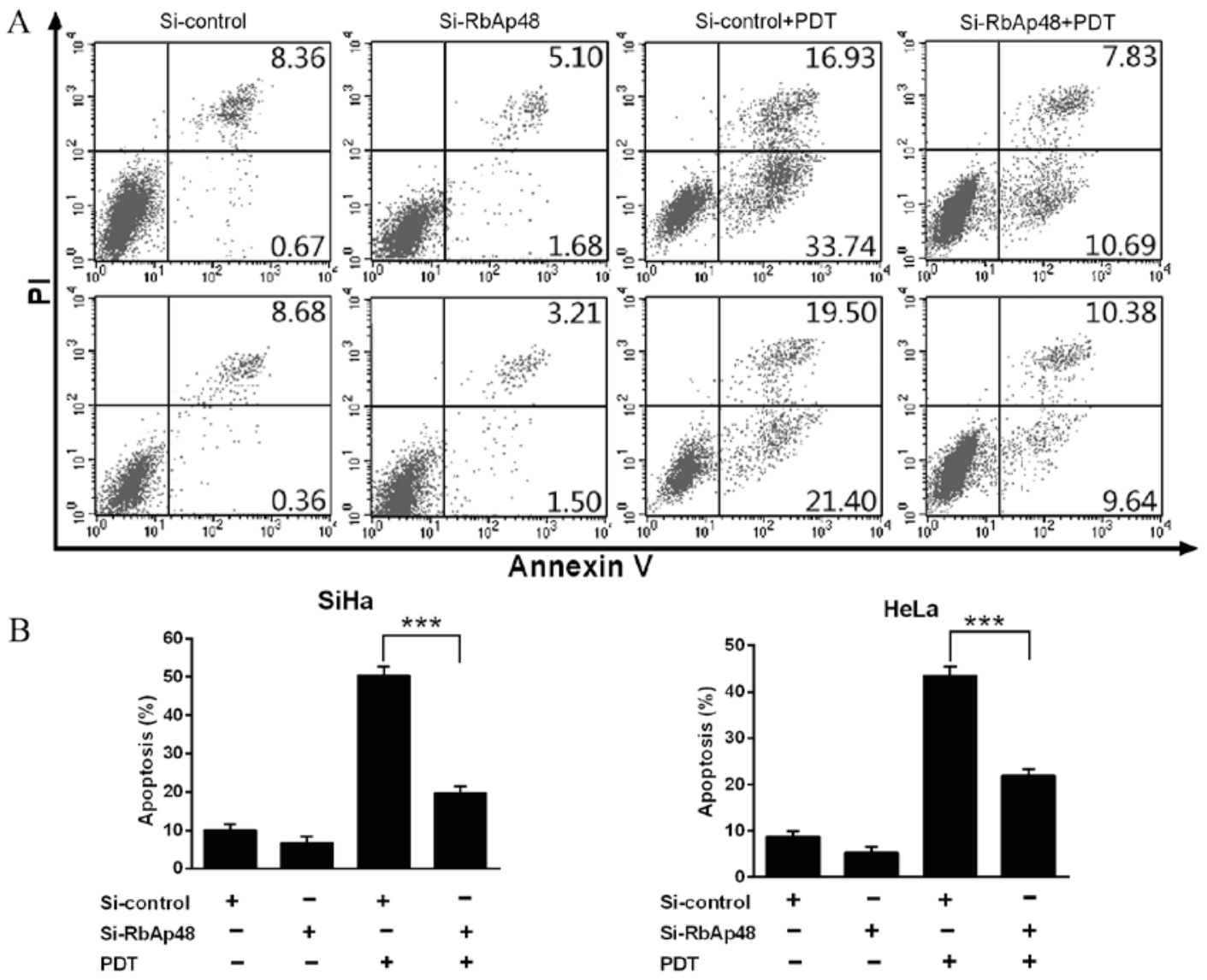

Figure 3. Reduced expression of RbAp48 in cervical cancer cell lines inhibits cell apoptosis induced by 5-aminolevulinic acid-mediated PDT. (A) Levels of apoptosis were assayed by flow cytometric analysis by the Annexin-fluorescein isothiocyanate/PI double staining method. Apoptotic cells were defined as Annexin V-FITC-positive cells. The Annexin V-FITC-positive and PI-negative area represents early apoptosis; the Annexin V-FITC- and PI-positive area represents end-stage apoptosis and death. The figure is representative of 3 independent experiments. (B) Percentage of apoptotic cells. Data are presented as the mean + standard deviation from 3 independent experiments. ( ${ }^{* * *} \mathrm{P}<0.001$ vs. si-control group). PI, propidium iodide; Si, small interfering RNA; RbAp48, retinoblastoma-associated protein 48 ; PDT, photodynamic therapy.

further ascertain the function of RbAp48 in PDT-induced cell destruction, targeted siRNA was used to reduce the expression of RbAp48 in SiHa and HeLa cells. Subsequently, cells from both cell lines were subjected to ALA-PDT and viability was examined. Reduced expression of RbAp48 in cervical cancer cells suppressed the decrease in cell viability usually induced by ALA-PDT, which confirmed that RbAp48 is an important contributor to PDT-mediated cell destruction.

Cancer is defined as abnormal cell growth characterized by increased cell proliferation and decreased apoptosis. With exposure to PDT, cells initiate complex responses, including the formation of highly reactive singlet oxygen or other free radicals like hydrogen peroxides or superoxide anions, which can kill tumor cells directly by apoptosis and/or necrosis $(10,23)$. The finding that PDT may cause an apoptotic response in target cells without complete disruption of the tissue has provided an explanation for the ideal characteristics required of any ablative technology for focally treating early cervical cancer. Certain studies have illustrated that apoptosis is critical for the therapeutic efficacy of PDT $(24,25)$ and reduced expression of RbAp48 inhibited apoptosis in exocrine gland cells (26). The current study investigated the effect of reduced RbAp48 on apoptosis, induced by PDT, which was assessed by flow cytometric analysis using the Annexin V-fluorescein isothiocyanate/propidium iodide double staining method. As presented in Fig. 3, reduced expression of RbAp48 in cervical cancer cells decreased PDT-induced apoptosis. It is therefore possible that PDT-mediated induction of RbAp48, at least partially, contributes to subsequent apoptosis of treated cells in addition to its inhibitory effect in malignant transformation, and its ability to enhance the radiosensitivity of cervical cancer cells. However, detailed studies are required to strengthen this hypothesis.

It is clear that p53 is associated with several types of human cancers and has great significance in cancer therapy. Studies have demonstrated that p53 has an important role in ALA-PDT-induced apoptosis in the HepG2 human liver cancer cell line (27) and the sensitivity of mutated p53 HT29 human colon adenocarcinoma cells to PDT can be increased by introducing the wild-type p53 gene (28). Rb is capable of suppressing cancer growth, partly by binding and inhibiting the activity of the E2F family of transcription factors (29-31). A previous study demonstrated that PDT increased the amount of hypo-phosphorylated $\mathrm{Rb}$ protein and decreased the amount of hyper-phosphorylated $\mathrm{Rb}$ protein in A431 cells, and suggested that $\mathrm{Rb}-\mathrm{E} 2 \mathrm{~F}$ was involved in PDT-induced cell cycle arrest and apoptosis (32). The current study reported that reduced RbAp48, combined with ALA-PDT, reduced mRNA levels of 
A

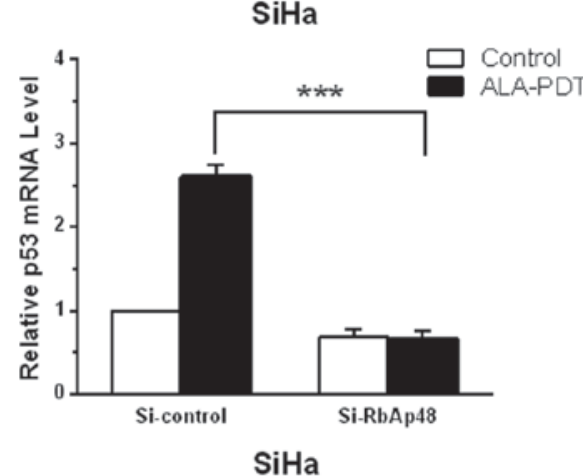

B

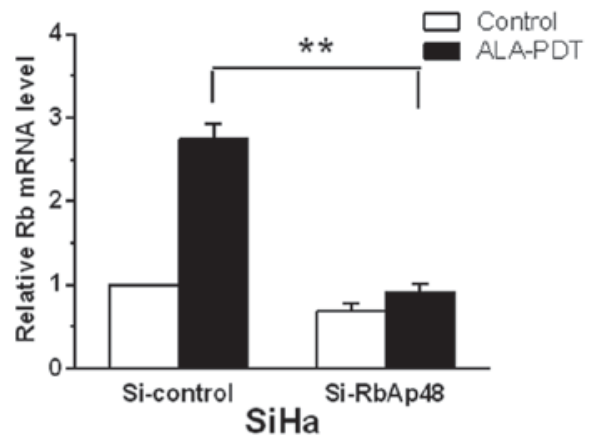

$\mathrm{C}$

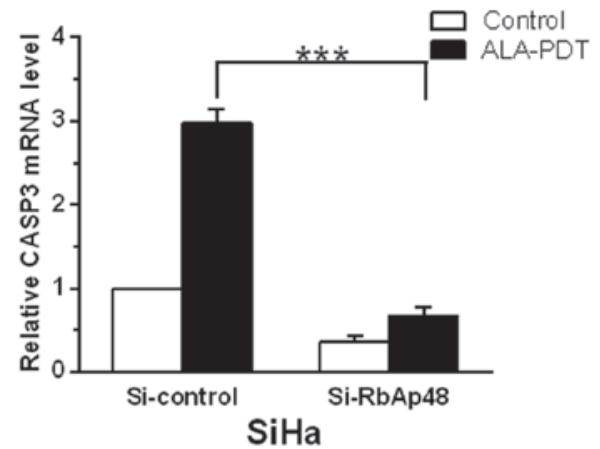

$\mathrm{D}$

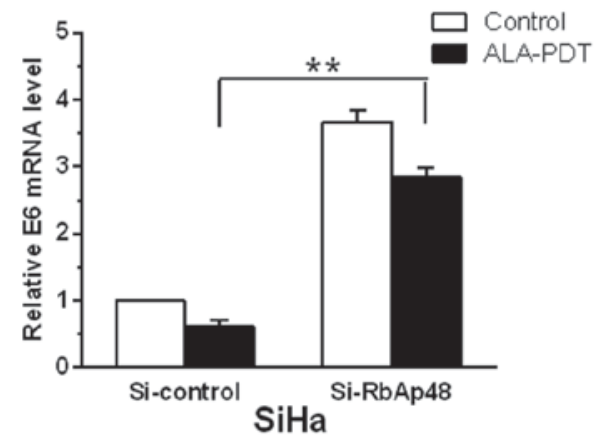

E

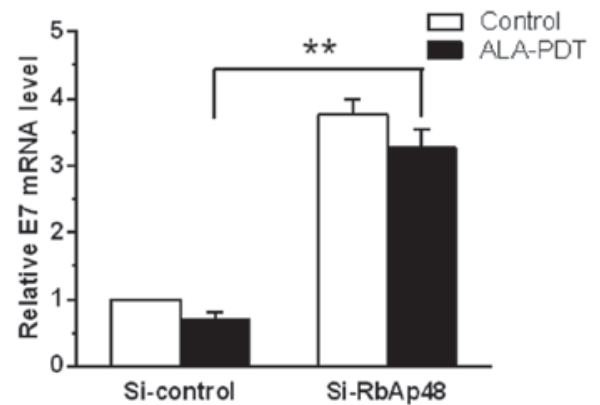

HeLa

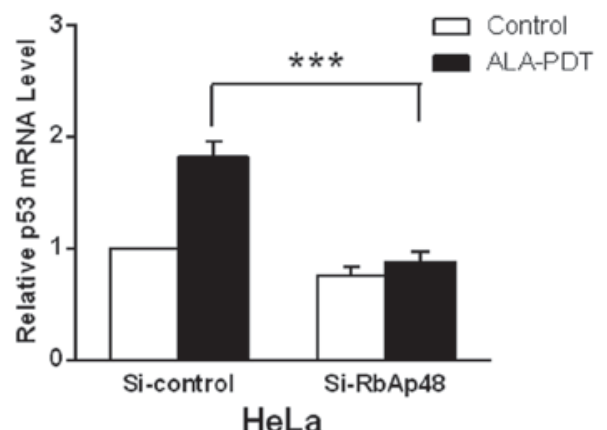

HeLa
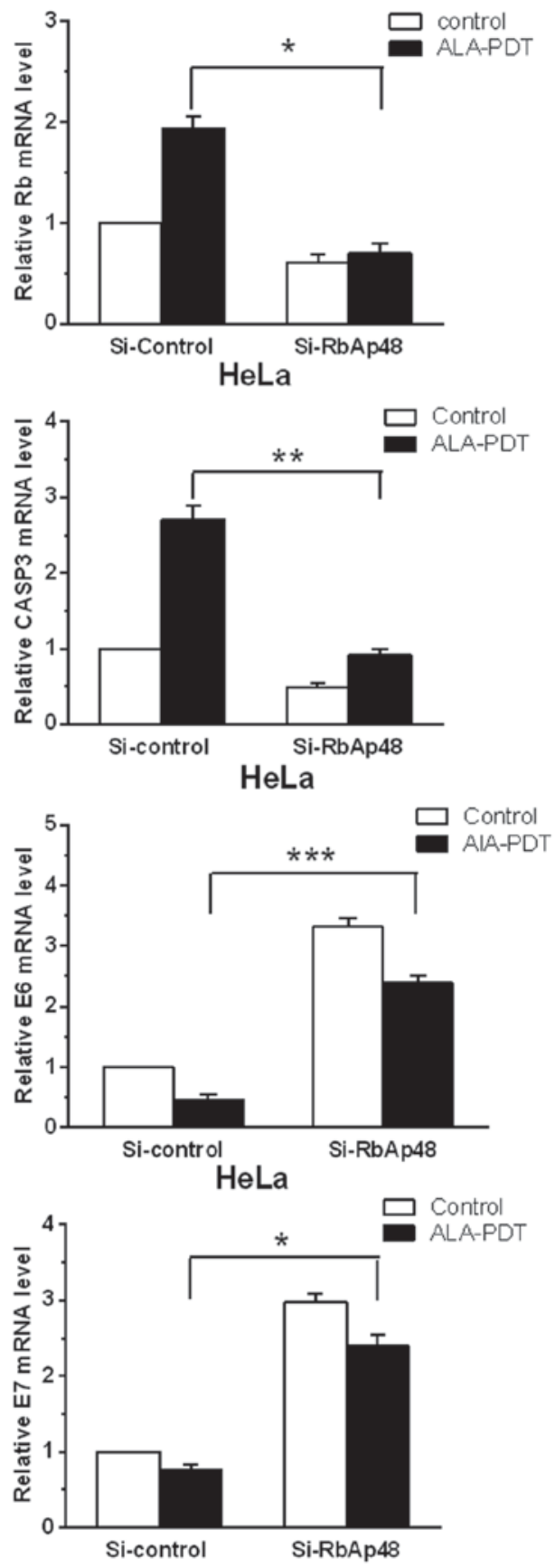

Figure 4. siRNA knockdown of RbAp48 regulates mRNA expression of p53, Rb, CASP3, E6 and E7 following ALA-PDT in cervical cancer cells. mRNA expression of (A) p53, (B) Rb, (C) CASP3, (D) E6 and (E) E7, in SiHa and HeLa cells with RbAp48 knockdown and/or ALA-PDT were assayed by reverse transcription-quantitative polymerase chain reaction. Expression of p53, Rb, CASP3, E6 and E7 in each sample was normalized to that of $\beta$-actin. Normalized values were then calibrated against si-control transfected values that were arbitrarily set as 1 . Quantification results are presented in the form of bar graphs. Data are presented as the mean \pm standard deviation from 3 independent experiments. $\left({ }^{*} \mathrm{P}<0.05 ;{ }^{* *} \mathrm{P}<0.01 ;{ }^{* * *} \mathrm{P}<0.001\right.$ vs. si-control group). p53, tumor suppressor protein 53; ALA-PDT, 5-aminolevulinic acid-mediated photodynamic therapy; Si, small interfering RNA; RbAp48, Retinoblastoma-associated protein 48; Rb, retinoblastoma protein; ALA, 5-aminolevulinic acid; CASP3, caspase-3. 
p53 and Rb in cervical cancer cells, compared with PDT treatment alone. Caspases are critical mediators of apoptosis (33). Reports have indicated that caspase- 3 is a death protease that is frequently activated during PDT-induced apoptosis, and is part of the mitochondria-initiated cytochrome c-mediated caspase-dependent pathway $(34,35)$. The present study demonstrated that RbAp48 altered the expression of caspase- 3 in cervical cancer cells in response to ALA-PDT.

It is well established that E6 and E7 are major viral oncogenes that promote host cell proliferation and decreased cell apoptosis, and increase genomic instability to facilitate the migration of HPV-infected cells (36). E6 and E7 proteins form complexes with p53 and $\mathrm{Rb}$, respectively, which leads to dysregulation of cell cycle control mechanisms and the release of transcription factor E2F, which then activates the expression of cell cycle-associated genes. Viral oncogenes E6 and E7 are essential for HPV-induced cervical carcinogenesis and support the viral life cycle (37). As RbAp48 controls the transforming activity of HPV-16 in cervical carcinogenesis by affecting mRNA levels of E6 and E7, the present study conducted RT-qPCR to investigate the effect of altered RbAp48 on E6 and E7 in cervical cancer cells that had been subjected to PDT. The results indicate that ALA-PDT effectively decreased mRNA levels of E6 and E7. Silencing RbAp48 increased mRNA levels of E6 and E7 in cervical cancer cells compared with cervical cancer cells subjected to ALA-PDT alone. It is therefore possible that the regulation of $\mathrm{p} 53$ and $\mathrm{Rb}$, caspase-3, and E6 and E7, by RbAp48 contributes, at least in part, to the cell destruction caused by PDT.

In conclusion, the results of the present study indicated that RbAp48 is involved in the anti-proliferative effects and apoptosis induced by PDT in cervical cancer cells. As earlier studies demonstrated that reduced expression of RbAp48 is specific to cervical cancer, and considering the important role RbAp48 has in regulating cervical carcinogenesis, measuring RbAp48 expression may have the potential to predict the response that individual cervical cancers will have to ALA-PDT. However, a detailed study to investigate the involvement of RbAp48 in PDT-mediated cell destruction, and the association with the expression of RbAp48 and PDT-mediated therapeutic efficacy in cervical cancer and its precancerous form, $\mathrm{CIN}$, are required to valiadate these findings.

\section{Acknowledgements}

The present study was supported by the National Natural Science Foundation of China (grant no. 81572559) and the Foundation for Outstanding Young Scientist of Shandong Province (grant no. BS2010YY056).

\section{References}

1. Ferlay J, Soerjomataram I, Ervik M, Dikshit R, Eser S, Mathers C, Rebelo M, Parkin DM, Forman D and Bray F: GLOBOCAN 2012 v1.0, cancer incidence and mortality worldwide: IARC CancerBase No. 11 [internet]. International Agency for Research on Cancer, Lyon. http://globocan.iarc.fr. Accessed December 2013.

2. Li S, Hu T, Lv W, Zhou H, Li X, Yang R, Jia Y, Huang K, Chen Z, Wang $S$, et al: Changes in prevalence and clinical characteristics of cervical cancer in the People's Republic of China: A study of 10,012 cases from a nationwide working group. Oncologist 18: 1101-1107, 2013.
3. Shishkova N, Kuznetsova O and Berezov T: Photodynamic therapy for gynecological diseases and breast cancer. Cancer Biol Med 9: 9-17, 2012.

4. Yu CH and Yu CC: Photodynamic therapy with 5-aminolevulinic acid (ALA) impairs tumor initiating and chemo-resistance property in head and neck cancer-derived cancer stem cells. PLoS One 9: e87129, 2014.

5. Trushina OI, Novikova EG, Sokolov VV, Filonenko EV, Chissov VI and Vorozhtsov GN: Photodynamic therapy of virus-associated precancer and early stages cancer of cervix uteri. Photodiagnosis Photodyn Ther 5: 256-259, 2008.

6. Choi MC, Kim MS, Lee GH, Jung SG, Park H, Joo WD, Lee C, Lee JH, Hwang YY and Kim SJ: Photodynamic therapy for premalignant lesions of the vulva and vagina: A long-term follow-up study. Lasers Surg Med Jul 14, 2015 (Epub ahead of print).

7. Saini R and Poh CF: Photodynamic therapy: A review and its prospective role in the management of oral potentially malignant disorders. Oral Dis 19: 440-451, 2013.

8. Soergel P and Hillemanns P: Photodynamic therapy for intraepithelial neoplasia of the lower genital tract. Photodiagnosis Photodyn Ther 7: 10-14, 2010.

9. Dewaele M, Martinet W, Rubio N, Verfaillie T, de Witte PA, Piette $\mathrm{J}$ and Agostinis P: Autophagy pathways activated in response to PDT contribute to cell resistance against ROS damage. J Cell Mol Med 15: 1402-1414, 2011.

10. Postiglione I, Chiaviello A and Palumbo G: Enhancing photodynamyc therapy efficacy by combination therapy: Dated, current and oncoming strategies. Cancers (Basel) 3: 2597-2629, 2011.

11. Demyanenko SV, Uzdensky AB, Sharifulina SA, Lapteva TO and Polyakova LP: PDT-induced epigenetic changes in the mouse cerebral cortex: A protein microarray study. Biochim Biophys Acta 1840: 262-270, 2014.

12. Nicolas E, Morales V, Magnaghi-Jaulin L, Harel-Bellan A, Richard-Foy $\mathrm{H}$ and Trouche D: RbAp48 belongs to the histone deacetylase complex that associates with the retinoblastoma protein. J Biol Chem 275: 9797-9804, 2000.

13. Zhang Y, Ng HH, Erdjument-Bromage H, Tempst P, Bird A and Reinberg D: Analysis of the NuRD subunits reveals a histone deacetylase core complex and a connection with DNA methylation. Genes Dev 13: 1924-1935, 1999.

14. Kong L, Yu XP, Bai XH, Zhang WF, Zhang Y, Zhao WM, Jia JH, Tang W, Zhou YB and Liu CJ: RbAp48 is a critical mediator controlling the transforming activity of human papillomavirus type 16 in cervical cancer. J Biol Chem 282: 26381-26391, 2007.

15. Torres-Roca JF, Eschrich S, Zhao H, Bloom G, Sung J, McCarthy S, Cantor AB, Scuto A, Li C, Zhang S, et al: Prediction of radiation sensitivity using a gene expression classifier. Cancer Res 65: 7169-7176, 2005.

16. Zheng L, Tang W, Wei F, Wang H, Liu J, Lu Y, Cheng Y, Bai X, Yu X and Zhao W: Radiation-inducible protein RbAp48 contributes to radiosensitivity of cervical cancer cells. Gynecol Oncol 130: 601-608, 2013.

17. Livak KJ and Schmittgen TD: Analysis of relative gene expression data using real-time quantitative PCR and the 2(-Delta Delta C(T)) Method. Methods 25: 402-408, 2001.

18. Soergel P, Loehr-Schulz R, Hillemanns M, Landwehr S, Makowski L and Hillemanns P: Effects of photodynamic therapy using topical applied hexylaminolevulinate and methylaminolevulinate upon the integrity of cervical epithelium. Lasers Surg Med 42: 624-630, 2010 .

19. Choi MC, Jung SG, Park H, Lee SY, Lee C, Hwang YY and Kim SJ: Photodynamic therapy for management of cervical intraepithelial neoplasia II and III in young patients and obstetric outcomes. Lasers Surg Med 45: 564-572, 2013

20. Ahn TG, Lee BR, Kim JK, Choi BC and Han SJ: Successful full term pregnancy and delivery after concurrent chemo-photodynamic therapy (CCPDT) for the uterine cervical cancer staged 1B1 and 1B2: Preserving fertility in young women. Gynecol Oncol Case Rep 2: 54-57, 2012.

21. Jerjes W, Upile T, Betz CS, El Maaytah M, Abbas S, Wright A and Hopper C: The application of photodynamic therapy in the head and neck. Dent Update 34: 478-480, 483-484, 486, 2007.

22. Hopper C: Photodynamic therapy: A clinical reality in the treatment of cancer. Lancet Oncol 1: 212-219, 2000.

23. Douillard S, Rozec B, Bigot E, Aillet L and Patrice T: Secondary reactive oxygen species production after PDT during pulmonary tumor growth in sera of nude mice. Photodiagnosis Photodyn Ther 10: 62-71, 2013. 
24. Gupta S, Dwarakanath BS, Muralidhar K and Jain V: Role of apoptosis in photodynamic sensitivity of human tumour cell lines. Indian J Exp Biol 41: 33-40, 2003.

25. Ke MS, Xue LY, Feyes DK, Azizuddin K, Baron ED, McCormick TS, Mukhtar H, Panneerselvam A, Schluchter MD, Cooper KD, et al: Apoptosis mechanisms related to the increased sensitivity of Jurkat T-cells vs A431 epidermoid cells to photodynamic therapy with the phthalocyanine Pc 4. Photochem Photobiol 84: 407-414, 2008.

26. Ishimaru N, Arakaki R, Omotehara F, Yamada K, Mishima K, Saito I and Hayashi Y: Novel role for RbAp48 in tissue-specific, estrogen deficiency-dependent apoptosis in the exocrine glands. Mol Cell Biol 26: 2924-2935, 2006.

27. Yow CM, Wong CK, Huang Z and Ho RJ: Study of the efficacy and mechanism of ALA-mediated photodynamic therapy on human hepatocellular carcinoma cell. Liver Int 27: 201-208, 2007.

28. Mikes J, Koval' J, Jendzelovský R, Sacková V, Uhrinová I, Kello M, Kuliková L and Fedorocko P: The role of p53 in the efficiency of photodynamic therapy with hypericin and subsequent long-term survival of colon cancer cells. Photochem Photobiol Sci 8: 1558-1567, 2009.

29. Weintraub SJ, Chow KN, Luo RX, Zhang SH, He S and Dean DC: Mechanism of active transcriptional repression by the retinoblastoma protein. Nature 375: 812-815, 1995.

30. Goodrich DW: The retinoblastoma tumor-suppressor gene, the exception that proves the rule. Oncogene 25: 5233-5243, 2006.
31. Nevins JR: The Rb/E2F pathway and cancer. Hum Mol Genet 10: 699-703, 2001.

32. Ahmad N, Gupta S and Mukhtar H: Involvement of retinoblastoma $(\mathrm{Rb})$ and E2F transcription factors during photodynamic therapy of human epidermoid carcinoma cells A431. Oncogene 18: 1891-1896, 1999.

33. Porter AG and Jänicke RU: Emerging roles of caspase-3 in apoptosis. Cell Death Differ 6: 99-104, 1999.

34. Furre IE, Møller MT, Shahzidi S, Nesland JM and Peng Q: Involvement of both caspase-dependent and -independent pathways in apoptotic induction by hexaminolevulinate-mediated photodynamic therapy in human lymphoma cells. Apoptosis 11: 2031-2042, 2006.

35. Fukuhara H, Inoue K, Kurabayashi A, Furihata M, Fujita H, Utsumi K, Sasaki J and Shuin T: The inhibition of ferrochelatase enhances 5-aminolevulinic acid-based photodynamic action for prostate cancer. Photodiagnosis Photodyn Ther 10: 399-409, 2013.

36. Münger K, Phelps WC, Bubb V, Howley PM and Schlegel R: The E6 and E7 genes of the human papillomavirus type 16 together are necessary and sufficient for transformation of primary human keratinocytes. J Virol 63: 4417-4421, 1989.

37. Govan VA: Strategies for human papillomavirus therapeutic vaccines and other therapies based on the E6 and E7 oncogenes. Ann N Y Acad Sci 1056: 328-343, 2005. 